\title{
Computational Method to Verify Static Alignment of Transtibial Prosthesis
}

\author{
Esperanza Camargo, Enrique Garzón and Lely A Luengas-C* \\ University Francisco Jose de Calda, Colombia \\ Corresponding author: Lely A Luengas-C, University Francisco Jose de Calda, Colombia
}

\section{ARTICLE INFO}

Received: 慧 October 01, 2020

Published: October 19, 2020

Citation: Esperanza Camargo, Enrique Garzón, Lely A Luengas-C. Computational Method to Verify Static Alignment of Transtibial Prosthesis. Biomed J Sci \& Tech Res 31(2)-2020. BJSTR. MS.ID.005074.

Keywords: Prosthetic Alignment, Transtibial Prosthesis, Neural Network

\section{ABSTRACT}

In Colombia, amputations are not only due to cardiovascular or traumatic causes. According to the Presidential Program for Action against Antipersonnel Mines, amputations inflicted by this type of mines increased between 1990 and 2020 due to the predominance of the armed conflict (mainly in the lower members). The most used method for the amputee is the prosthesis, however, the prosthetic adaptation requires processes and procedures that go according to each individual, existing one generalized, the alignment of the prosthesis. Alignment is the key element of an optimal prosthetic function. The relationship between the prosthesis, the patient, and the components of the prosthesis, critically affects the functional performance and comfort. A new static alignment method for transtibial prostheses is suggested using computational models. An application was made in MATLAB®environment, making use of Neural Networks of Generalized Regression (GRNN), which allows predicting biomechanical data from the alignment of the prosthesis socket. A graphical user interface was developed to show the functioning of the algorithm, this was validated with recorded data of amputees, which allowed to evaluate the repeatability and accuracy of the results obtained. This application seeks to build a support system for the prosthetics technician to improve the rehabilitation process of a person using transtibial prosthesis.

\section{Introduction}

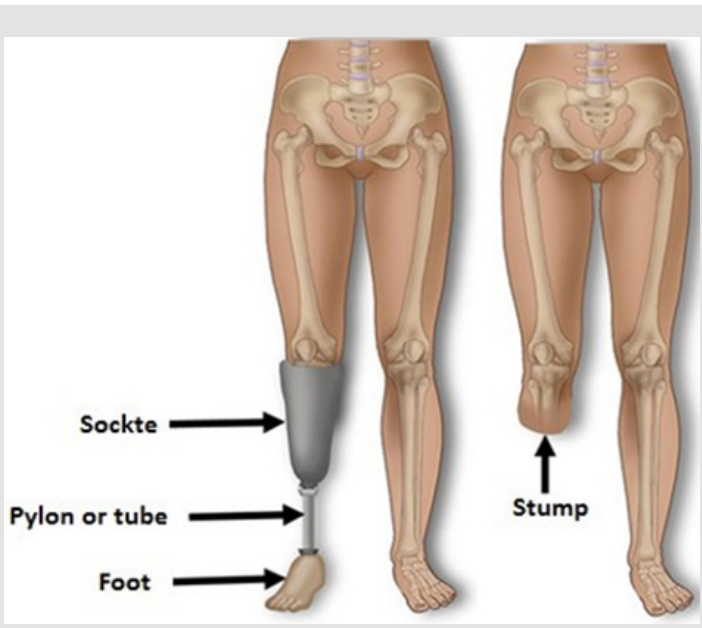

Figure 1: Transtibial prosthesis and components.

The incidence of amputation worldwide is 1.5 per 1000 habitants and the total number of amputees is about 10 million people in the world. In Colombia, the number of amputees is about
46,200 , transtibial amputation being the most common, mainly caused by anti-personnel mines. The Presidential Program for Integral Action against Mines, dependency of the Administrative Department of the Presidency of the Republic reports that between 1990 and July 2020 there has been 11,993 victims bymines, 2202 lost their lives and 8791 suffered injuries[1].For the rehabilitation, the amputee commonly uses prosthesis, which in transtibial amputation it is called transtibial prosthesis. The part of the limb that remains after an amputation is called a stump. The interface between the prosthesis and the stump is the socket (socket), Figure 1 [2].

The adaptation of the prosthesis to the individual is one of the important aspects to be taken into account during the process of prosthetic rehabilitation. The alignment of the prosthesis allows to properly locate both of the components with each other, as well as the entire prosthesis to the patient. The alignment is one of the determining factors to assure comfort to the user and in the fulfilment of its function on limb replacement. For this reason, 
making changes in the alignment can have important consequences on the biomechanical and physiological efficiency of walking and other activities of the user's daily life, the gravitational vertical is displaced by altering the load distribution, Figure 2 [3,4].Despite of the evident importance of this parameter, the tendency in the medical field is to perform the alignment by an observational method, based on the expertise of the medical personnel who perform the alignment, and trial and error techniques, leading to empirical and subjective results[5-7].

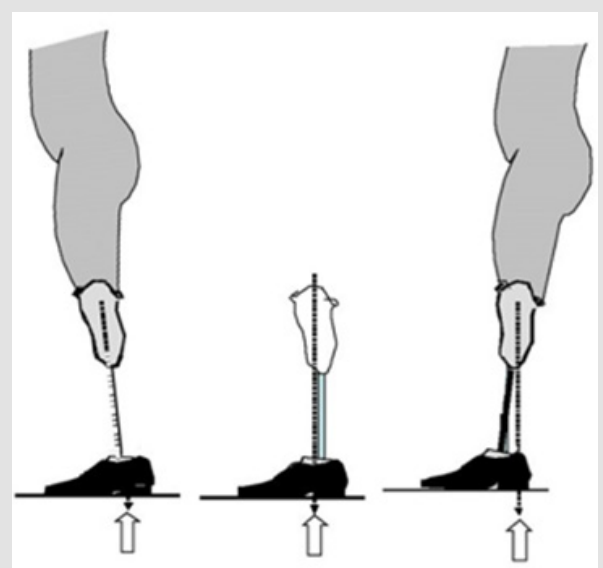

Figure 2: Different locations of the socket and the involvement in the line of action of gravity on the stump.

Manufacturers of prostheses give static alignment recommendations from clinical experience, these general guidelines do not reflect an objective method. It requires technological tools that allow an ideal alignment, easy to use and easy to record the biomechanical parameters of the amputees. The possibility of applying computational methods to prosthetic alignment is the subject of this paper. Therefore, an algorithm based on Generalized Regression Neural Networks (GRNN) was used to predict the location of the pressure centre (COP) and the joint ranges of the lower segments of the body according to the position angle of the prosthesis socket.The GRNN allows modelling unknown relationships and non-linear nature, this type of network is used to develop regressions between dependent and independent variables, and it consists of an input layer, hidden layers and an output layer, Figure 3. They are based on normalized Radial Base Functions Networks (RBF), where there is a cell in the hidden layer corresponding to each training pattern. The algorithm of neural networks makes use of the Bayes-Parzen classifier and its architecture in the probabilistic networks or functions of Radial Base, so this type of networks is based on the Theory of Nonlinear Regression, its estimation mechanism makes use of Kernel density estimation. The GRNN do not require iterative training, they canapproximate any arbitrary function between input vectors and outputs making the estimation of the function directly from the training data. In addition, they have the property of consistency as the training set increases; the estimation error tends to zero, with only moderate restrictions on the function.

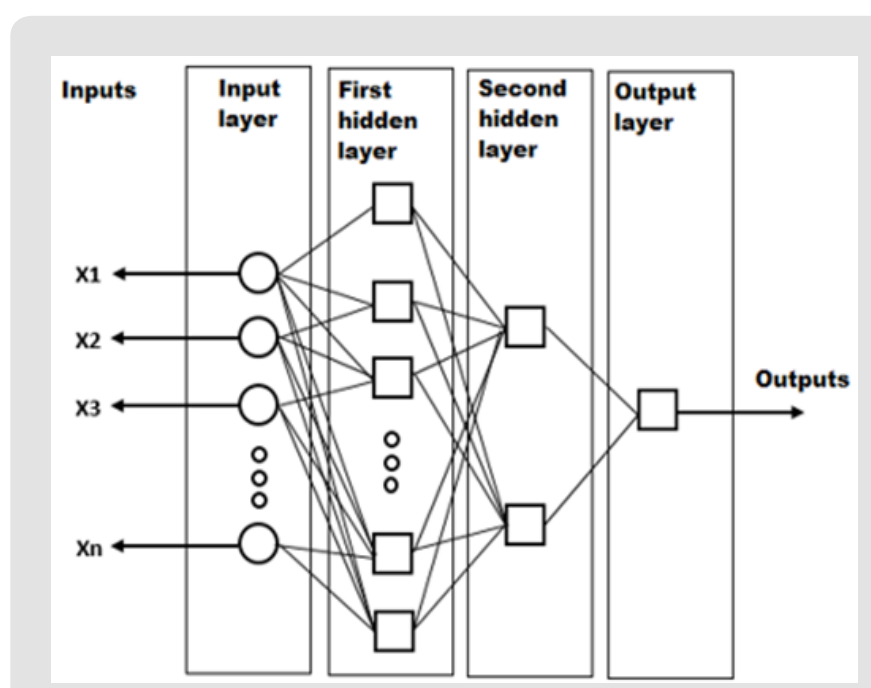

Figure 3: Model of a Neural Network of Generalized Regression (GRNN)

It is possible to consider the GRNN as a universal approximation for smooth functions. The fundamental expression of the neuronal model of generalized regression (GRNN), is given in

$$
\hat{y}=\frac{N(X)}{D(X)}=\frac{\sum_{i=1}^{n} Y_{i} \cdot e^{\left(-D\left(X, X_{1}\right)\right)}}{\sum_{i=1}^{n} e^{\left(-D\left(x, x_{1}\right)\right)}}
$$

Eq (1) [8-10].Where, $y_{i}$ corresponds to the estimation of the outputs and $\mathrm{D}\left(\mathrm{X}, \mathrm{X}_{\mathrm{i}}\right)$ to the distance between the vector of inputs (X) and the i-th reference vector.This application seeks to build a support system for the prosthesis technician to improve the rehabilitation process of a person using transtibial prosthesis, based on the use of computational tools that show the relationship between the data of a patient's position against a patient model that shows the correct adjustment parameters.

\section{Methods}

A computational model of the protocol that allowed the integral registration of clinical data, anthropometry and biomechanical data was designed as a proposal. We recruited an unilateral transtibial amputee left side user of modular endoskeleton prosthesis for more than 7 years, functional in standing and walking without external aids, with a normal range of mobility of the joints of the lower limbs, with prosthetic alignment certified by prosthesis. The type of prosthesis is with suspension by liner and pin and foot in high activity carbon fibre. The evaluation of the study participant was made by a doctor, observing that there was no inflammation, with ulcers or any other pathology in the stump, that reduced the reliability of the samples.Initially, measurements of biomechanical parameters such as angle of hip, knee and ankle were taken both 
on the amputated (ipsilateral) and non-amputated (contralateral) sides, distribution of plantar forces and location of the COP, for this the Pedar® systems were used (Novel, Germany) for data collection of the distribution of plant forces and location of the COP[10]; and BiomectricsLtda ${ }^{\circledR}[11]$ for the measurement of the angles of the joint and socket segments[11]. The first measurement was made with the prosthesis in alignment. The second measurement was made by varying the angular location of the socket, it was placed in flexion. The third measurement was with the socket in extension.

Then the data was analyzed, filtering for atypical data and obtaining measures of central tendency and distribution. The type of statistical distribution of the data was analysed. The angular location of the prosthesis socket was selected as the entrance to the GRNN. The biomechanical descriptive variables (joint ranges and COP in ipsi and contralateral) were selected as output from the network.The GRNN was designed with radial base neurons and a linear layer, with the following characteristics: 700 hidden layers (Data obtained), one Output layer (Approximate), one Input (Angle Variation).The input data is transferred to the neurons of the first hidden layer, which has a number of neurons determined by the number of cases that make up the database. The algorithm obtained was validated and evaluated based on the repeatability and accuracy of the results obtained. The training of the GRNN was done with the data of a voluntary subject and validated against data of the same subject making use of different statistical tools designed for that purpose. For the selection of training and validation data, cross-validation was used, which allows to randomly divide the data and form two groups: one of training and one of validation. An application was designed to predict the behaviour of the biomechanical parameters present in the static alignment of prosthesis of a transtibial amputee by prosthetic user antipersonnel mines, depending on the location of the prosthesis socket.

\section{Results and Discussion}

To observe the behavior of joint angles and COP, we affected the location of the socket. Then, the alignment directly affects the position of the lower body segments of the amputees; this affects the distribution of body burden, the reaction forces of the floor and the pressure center, misalignment may be contribute to the high incidence of osteoarthritis $[12,13]$.The data does not present a statistical distribution of normal type, they are multimodal. Therefore, statistical analysis should use non-parametric techniques $[14,15]$. Under all of the alignment conditions, the COP on the intact side were significantly larger than those on the residual side. The hip angle on the intact side were all larger than those on the residual side, but significant differences were found only under some of the alignment conditions. Other joins was similar behavior [16]. The results of the study showed that COP and join angles might well indicate the confidence and/or (dis) comfort of the amputee subjects to load the prosthetic leg and they are could be indicators of alignment $[17,18]$. The architecture of the model proved to be efficient by requiring a minimum processing time. In general, training sessions require less than 5 minutes reaching the objective training objectives. The maximum error of approximation of the GRNN was presented in the angle for the hip of the non-amputated side with an error of $6.25 \%$. The minimum error was 0.51 in the location of the COP direction Y.

Figure 4 shows the developed interface where the mass of the individual and the angular position of the socket of the prosthesis are entered as inputs, the outputs of the system are the hip, knee and ankle angles, position COP and distribution of force in each foot, this on both the amputated and the contralateral side. Additionally, it allows observing the response curves of each output depending on the value of the input angle.Figure 5 shows the relationship between the variation of the angle of the prosthesis and its reaction in the posture, as well as its behaviour during a controlled time range.

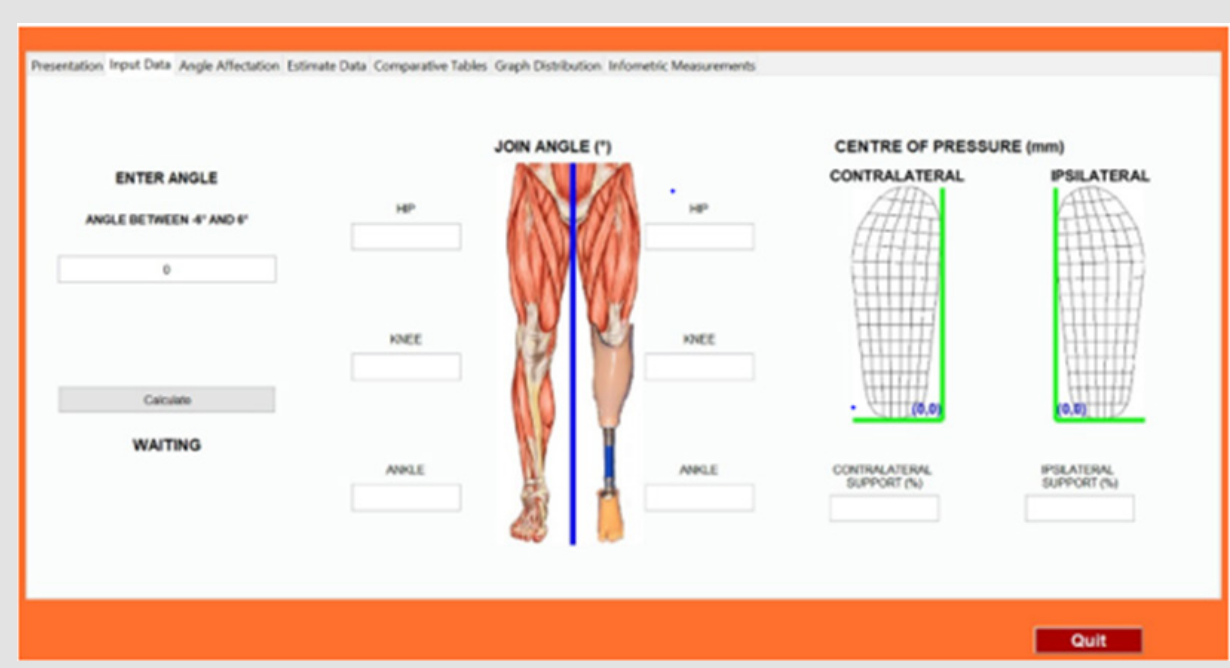

Figure 4: Interface of the GRNN as support in the static alignment of transtibial prostheses. 


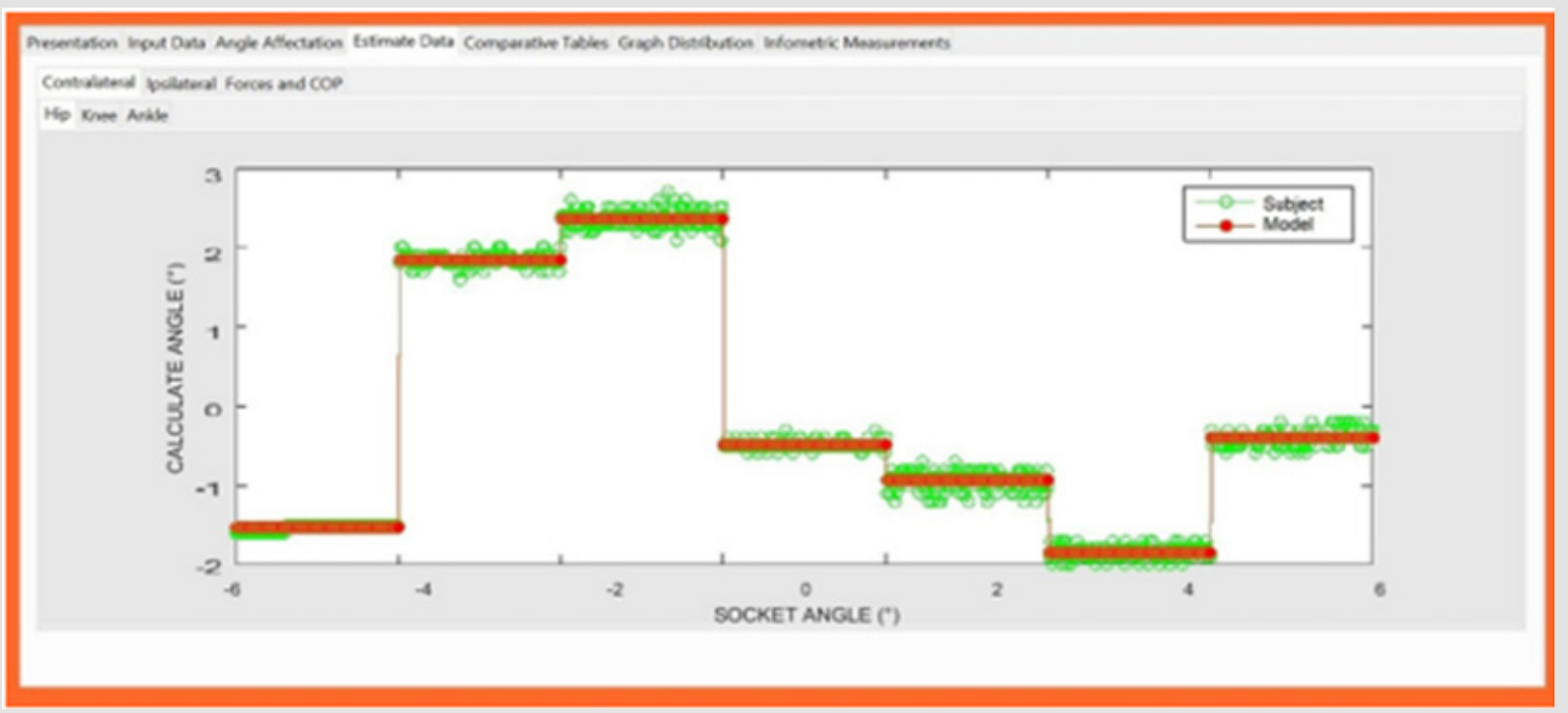

Figure 5: Angle of the hip contralateral side depending on the location of the socket.

\section{Conclusions}

According to the analysis of the data, a tendency to the average value can be observed in each measurement which means that there are many variations during the taking of the samples. The behaviour reflected on the limb will be the average value of the total samples obtained.This study demonstrated the ability of a GRNN model to accurately predict the behaviour of biomechanical parameters according to socket alignment in static standing position measurements (static alignment). The results supported the hypothesis that GRNN model architecture is adequate to estimate static alignment based on basic measurements of COP and joint ranges.The network designed and implemented reduced the dimensionality of the classification problem and made the processing of the data simpler. The GRNN allowed to predict the affectation of the selected biomechanical parameters, in the future other parameters can be added and observe how the alignment affects them.The error throws the system is greater than $1 \%$ in some points but with the help of the neural network that dispersion was reduced which allows to say that it is a reliable tool and can be used to know the values of the biomechanical parameters of standing in the static alignment of prostheses for transtibial amputees due to antipersonnel mines.

\section{References}

1. Biometrics Ltd (2020) Twin-Axis Goniometers for Dynamic Joint Movement Analysis. Retrieved

2. Blumentritt S, Schmalz T, Jarasch R (2001) Significance of static prosthesis alignment for standing and walking of patients with lower limb amputation. Der Orthopade 30(3): 161-168.

3. Boone DA, Kobayashi T, Chou TG, Arabian AK, Coleman KL, et al. (2012) Influence of malalignment on socket reaction moments during gait in amputees with transtibial prostheses. Gait \& Posture 37(4): 620-626.
4. Clemente YM, Roque DM (2013) Magnetic Resonance Brain Imaging Segmentation based on Generalized Regression Neural Networks. Cuban Journal of Medical Informatics 5(1): 82-90.

5. Dirección Contra Minas (2020) Victims of antipersonnel mines and unexploded ordnance.

6. Findlow A, Goulermas JY, Nester C, Howard D, Kenney LP J, et al. (2008) Predicting lower limb joint kinematics using wearable motion sensors. Gait \& Posture 28(1): 120-126.

7. Haykin S (1998) Neural Networks: A Comprehensive Foundation (2nd ed.). Patparganj: Pearson Education.

8. Jung Y, Jung M, Lee K, Koo S, Korea S, et al. (2012) Ground Reaction Force Estimation Using Musculoskeletal Simulation. 30th Annual Conference of Biomechanics in Sports (145): 389-392.

9. Lannon N (2004) Trans-tibial alignment. Static alignment. ORTHOLETTER (13): 11-13.

10. Luengas C LA, Camargo E, Guardiola D (2018) Modeling and Simulation of Prosthetic Gait Using a 3-D Model of Transtibial Prosthesis. Revista Ciencias de La Salud 16(1): 82-100.

11. Luengas C LA, Gutierrez MA, Camargo E (2017) Alignment of prostheses and biomechanical parameters of transtibial amputees. Bogota: UD Editorial.

12. Luengas C LA, Toloza DC (2020) Application of wavelet transform to stability analysis in transtibial amputees. Investigación e Innovación En Ingenierías 8(1): 214-225.

13. Nederhand MJ, Van Asseldonk EHF, Der Kooij H Van, Rietman HS (2012) Dynamic Balance Control (DBC) in lower leg amputee subjects; Contribution of the regulatory activity of the prosthesis side. Clinical Biomechanics 27(1): 40-45.

14. Novel de (2019) The pedar ${ }^{\circledR}$ system.

15. Póczos B (2009) Identification of Recurrent Neural Networks by Bayesian Interrogation Techniques. Journal of Machine Learning Research 10(18): 515-554.

16. Sarralde M (2020) Minas antipersonal y explosivos: balance del CICR sobre víctimas civiles y de fuerza pública en 2020. EL TIEMPO. 
Tafti N, Hemmati F, Safari R, Karimi MT, Farmani F, et al. (2018) A systematic review of variables used to assess clinically acceptable alignment of unilateral transtibial amputees in the literature. Journal

17. Tafti N, Karimlou M, Mardani MA, Jafarpisheh AS, Aminian GR, et al (2020). Development and preliminary evaluation of a new anatomically

ISSN: 2574-1241

DOI: $10.26717 /$ BJSTR.2020.31.005074

Lely A Luengas-C. Biomed J Sci \& Tech Res

(c) (i) This work is licensed under Creative

Submission Link: https://biomedres.us/submit-manuscript.php based prosthetic alignment method for below-knee prosthesis. Assistive Technology 32(1): 38-46.

18. Zhang T, Bai X, Liu F, Ji R, Fan Y, et al. (2020) The effect of prosthetic alignment on hip and knee joint kinetics in individuals with transfemoral amputation. Gait and Posture 76(1): 85-91.

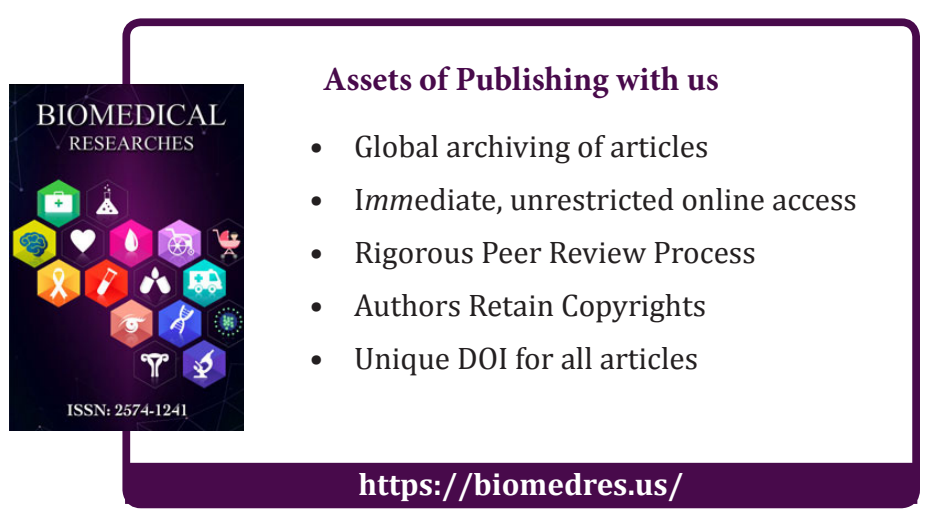

